Marquette University
e-Publications@Marquette

Master's Theses (2009 -)

Dissertations, Theses, and Professional

Projects

\title{
Efficacy of Invisalign Attachments: A Retrospective Study
}

Theresa Karras

Marquette University

Follow this and additional works at: https://epublications.marquette.edu/theses_open

Part of the Dentistry Commons

Recommended Citation

Karras, Theresa, "Efficacy of Invisalign Attachments: A Retrospective Study" (2019). Master's Theses

(2009 -). 536.

https://epublications.marquette.edu/theses_open/536 
Theresa Karras, DMD

A Thesis submitted to the Faculty of the Graduate School, Marquette University, in Partial Fulfillment of the Requirements for the Degree of Master of Science

Milwaukee, WI

August 2019 


\title{
ABSTRACT \\ EFFICACY OF INVISALIGN ATTACHMENTS: A RETROSPECTIVE STUDY
}

\author{
Theresa Karras, DMD
}

Marquette University, 2019

Objective: To compare the efficacy of Invisalign's (Align Technology, Inc., Santa Clara, CA) optimized and conventional attachments on rotational and extrusive tooth movements.

Materials and Methods: Initial, predicted, and achieved digital dental models from 100 orthodontic patients were exported from Invisalign's ClinCheck software as stereolithography (STL) files and subsequently imported into the Slicer CMF program (open source, version 4.7.0) for superimpositions on posterior teeth with no planned movement. Rotational and extrusive measurements for both optimized and conventional attachment types were made on 382 teeth from the superimposition of the initial and predicted models ("predicted movement") and from the superimposed initial and achieved models ("achieved movement"). Predicted and achieved movements were compared using paired $t$-tests. Independent $t$-tests were used to compare tooth movements for optimized versus conventional attachments. One-way ANOVAs were used to compare accuracies by tooth type. Data analysis was performed at the $P<0.05$ significance level.

Results: Mean differences between predicted and achieved movements were of high statistical significance for all attachment types and tooth movements. For extrusion, this mean difference was also clinically significant $(0.40 \mathrm{~mm}$ and $0.62 \mathrm{~mm}$ for optimized and conventional attachments, respectively). Overall mean accuracy was 57.2\%. Mean accuracy was $63.2 \%$ for rotation and $47.6 \%$ for extrusion. The most accurate tooth movement was extrusion of the maxillary central incisor with a conventional attachment $(75.9 \%)$ and the least accurate was extrusion of the mandibular canine with a conventional attachment (16.1\%). These differences were statistically significant. There was no significant difference in accuracy between tooth types for rotation. Interproximal reduction (IPR) or spacing did not significantly affect accuracy. Differences between accuracies of tooth movements using optimized versus conventional attachments for both rotation and extrusion were neither statistically nor clinically significant.

Conclusion: Conventional attachment types may be just as effective as Invisalign's proprietary optimized attachments for rotations of canines and premolars, and for extrusion of incisors and canines. Clinicians should consider overcorrection or midcourse correction, especially for extrusion of anterior teeth. A larger sample size of teeth with IPR or spacing is needed to draw more definitive conclusions about how these conditions affect the accuracy of tooth movements. 


\section{ACKNOWLEDGEMENTS}

\section{Theresa Karras, DMD}

My sincere appreciation to my advisor, Dr. Bhoomika Ahuja, for her time and guidance in this research and throughout my orthodontic education at Marquette University. This project would not have been brought to fruition without her support. I would like to thank the orthodontists, who wish to remain anonymous, for welcoming me to their private practices with open arms. By trusting me to access patient files and extract data, they undoubtedly helped build the foundation for this study. I would also like to thank Dr. Maharaj Singh for his knowledge and assistance in data analysis, and Emilia Karkazis for enthusiastically completing tasks vital to this project. Last but not least, I thank my family for their constant encouragement, unconditional love, and everlasting faith instilled in me throughout the years. I am truly grateful for them and for the path that God has chosen for me. 


\section{TABLE OF CONTENTS}

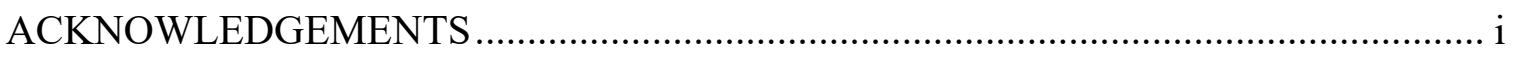

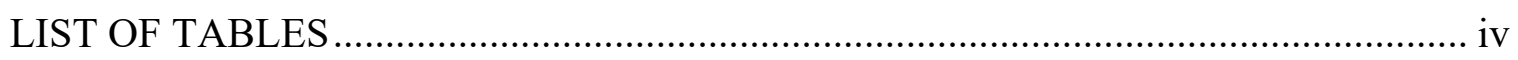

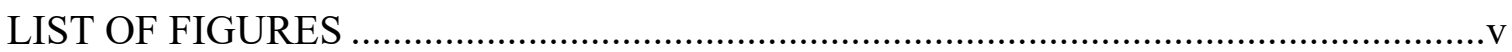

LIST OF ABBREVIATIONS ........................................................................... vi

CHAPTER

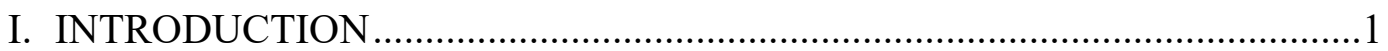

A. History of Orthodontic Appliances ..................................................

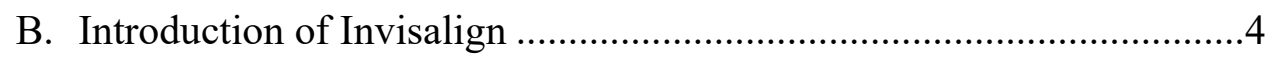

II. REVIEW OF LITERATURE .........................................................6

A. Invisalign Guidelines .............................................................6

B. SmartTrack Material ....................................................................6

C. Invisalign Attachments ...........................................................

D. Accuracy of Invisalign ......................................................... 10

E. Invisalign vs. Fixed Appliances ................................................. 14

III. OBJECTIVE AND HYPOTHESES .................................................... 15

IV. MATERIALS AND METHODS ....................................................... 16

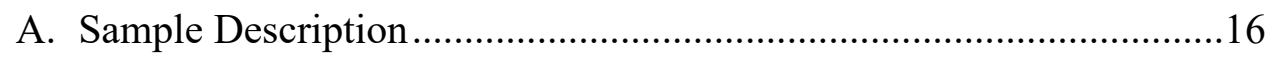

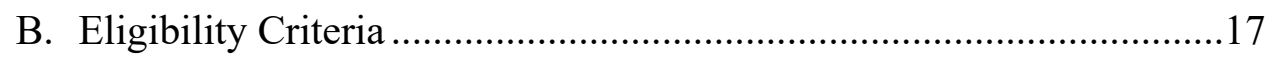

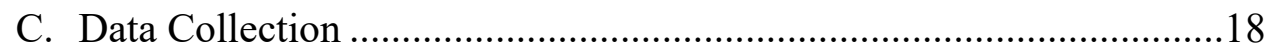

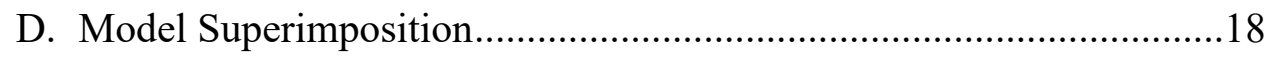

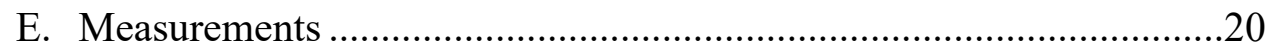

F. Statistical Analysis ............................................................ 21 


\section{TABLE OF CONTENTS (continued)}

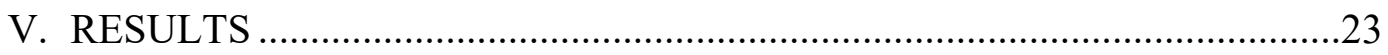

A. Intra-examiner Reliability ...........................................................23

B. Sample Characteristics.................................................................23

C. Predicted vs. Achieved Movements ..................................................25

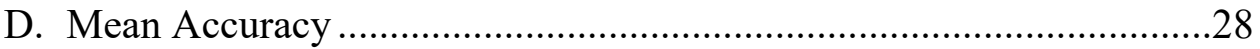

E. Optimized vs. Conventional Attachments .....................................30

F. Interproximal Reduction (IPR) and Spacing.................................32

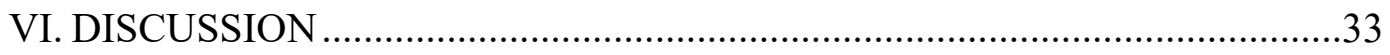

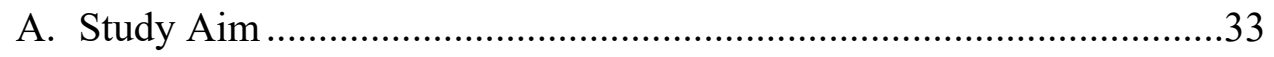

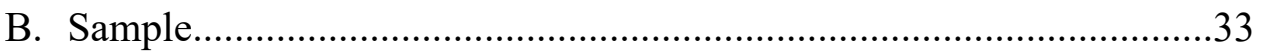

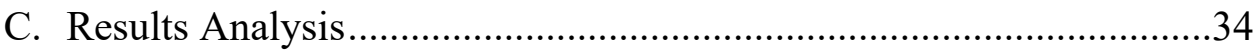

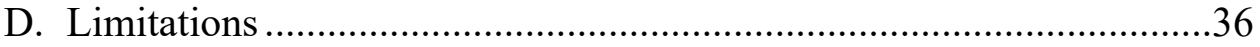

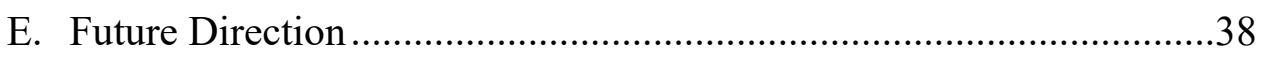

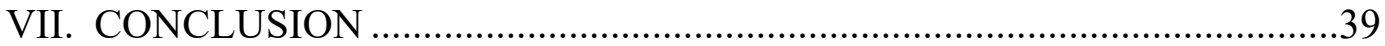

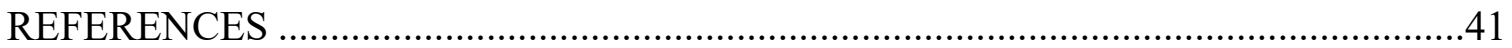




\section{LIST OF TABLES}

Table 1. Descriptive Statistics for Optimized Rotation Attachments ............................25

Table 2. Descriptive Statistics for Conventional Rotation Attachments........................26

Table 3. Descriptive Statistics for Optimized Extrusion Attachments...........................26

Table 4. Descriptive Statistics for Conventional Extrusion Attachments .....................27

Table 5. Predicted Movements vs. Achieved Movements ...........................................27

Table 6. ANOVA Comparing Mean Difference by Tooth Type .................................28

Table 7. Descriptive Statistics for Mean Accuracy …...............................................29

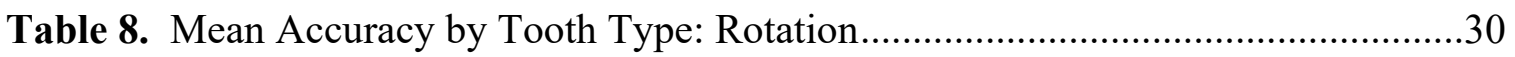

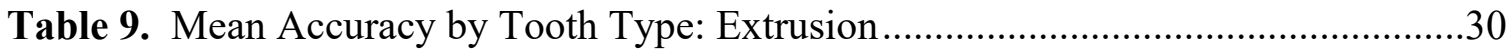

Table 10. ANOVA Comparing Mean Accuracy by Tooth Type ..................................30

Table 11. Optimized Attachments vs. Conventional Attachments $\left({ }^{\mathbf{0}}\right.$ and $\left.\mathrm{mm}\right)$...............31

Table 12. Mean Accuracy by Attachment Type and Tooth Movement.........................31

Table 13. Optimized Attachments vs. Conventional Attachments $(\%)$..........................31

Table 14. Mean Accuracy by Interproximal Condition ............................................32

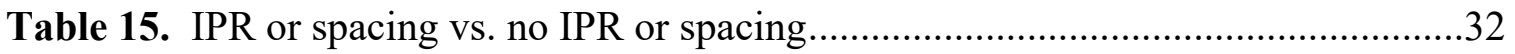




\section{LIST OF FIGURES}

Figure 1. The Invisalign Appliance ............................................................................

Figure 2. Conventional Rectangular Attachments ...................................................

Figure 3. Optimized Rotation Attachments ......................................................

Figure 4. Optimized Extrusion Attachments...........................................................

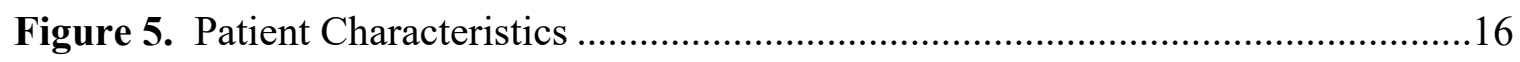

Figure 6. 3-D Model Superimposition Using 3D Slicer CMF ....................................19

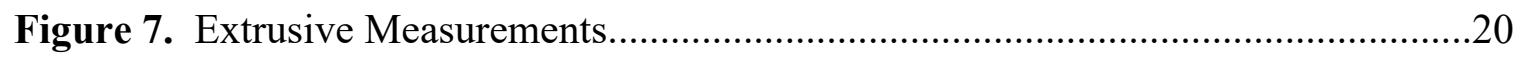

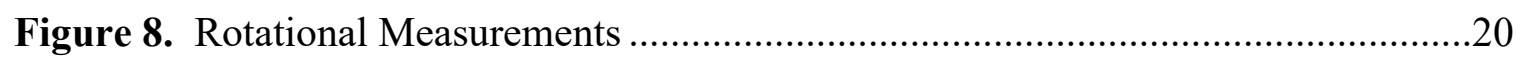

Figure 9. Tooth Movement Characteristics: Predicted Rotation..................................24

Figure 10. Tooth Movement Characteristics: Predicted Extrusion ...............................24 


\section{LIST OF ABBREVIATIONS}

3-D

$\mathrm{ABO}$

ANOVA

CI

ICC

IRB

IPR

ROI

SD

STL

USB
Three-Dimensional

American Board of Orthodontics

Analysis of Variance

Confidence Interval

Intraclass Correlation Coefficient

Institutional Review Board

Interproximal Reduction

Region of Interest

Standard Deviation

Stereolithography

Universal Serial Bus 


\section{CHAPTER I: INTRODUCTION}

\section{A. History of Orthodontic Appliances}

Orthodontics was the first recognized specialty of dentistry and involves the diagnosis and treatment of skeletal and dental malocclusions. Reasons patients may seek orthodontic care include to improve their current occlusion or oral function. However, according to William R. Proffit, author of the leading textbook for orthodontic education, "the major reason people seek orthodontic treatment is to minimize psychosocial problems related to their dental and facial appearance" (11). Over the years, there has been a progression towards more esthetic orthodontic appliances, as technology has further advanced and as more adults are seeking orthodontic treatment.

Orthodontic appliances consist of two types: fixed and removable. Fixed appliances are secured to teeth using temporary cements or composite bonding material and are not meant to be removed by the patient. Some examples of fixed appliances include traditional braces, expanders, and space maintainers. Removable appliances, such as clear aligners and headgear, can be detached by the patient and are only functioning when worn.

Fixed appliances used in modern orthodontics are based on Edward H. Angle's designs from the early twentieth century. Angle's original "edgewise" appliance, developed in 1928, featured metal brackets soldered to gold bands encircling each individual tooth. The brackets consisted of a horizontal rectangular slot and used stainless steel archwires to align teeth. Archwires were secured to the back of the bracket slot using steel ligatures ties. Tooth movements could be controlled in three dimensions 
by placing bends in a rectangular wire. These bends include: first-order, or in-out bends; second order, or mesiodistal tip; and third order to control torque, or labiolingual root angulation (Proffit et al. 358-60).

Today, these concepts are still used in the modern edgewise "twin bracket" system. However, instead of placing bands around each tooth, metal or ceramic brackets are bonded onto the facial or lingual surfaces of individual teeth. Furthermore, clinicians need to rely less on placing bends in wires to move individual teeth, as contemporary systems have "prescriptions" built into the bracket base or slot themselves, which become active when a preformed round or rectangular wire is inserted into the slot. This "straight wire" appliance was developed by Lawrence F. Andrews in the 1980s (Proffit et al. 361). Around this same time period, self-ligating, ceramic, and lingual brackets were also introduced.

Self-ligating brackets involve a clip attached to the bracket to secure the wire into place, and thus, eliminate the need for elastomeric ties, which take time to place and whose forces becomes reduced when stretched and exposed to the oral cavity (Proffit et al. 375). These brackets are divided into two main types: "active" and "passive," and both of these are made in metal or ceramic. Although it has been found that self-ligating brackets save some time in ligation, they do not reduce treatment time as a whole or produce better results (Proffit et al. 375).

Ceramic brackets were developed to overcome the esthetic limitations of metal braces. Although an excellent esthetic option, some limitations include: bulkiness, bracket fractures due to their brittle glass-like nature, friction within bracket slots (overcome when a hybrid bracket with a metal slot is used), enamel wear due to hardness, 
and potential enamel breakage when debonding (Proffit et al. 370). Lingual braces are virtually invisible, as they are custom-fit to the back surfaces of teeth. Aside from being almost unnoticeable, another advantage of these types of braces over labial appliances include reduction of white spot lesions, which are due to decalcification on enamel surfaces. Some disadvantages include difficulty in archwire insertion, discomfort during speaking and eating, and tongue irritation (Graber et al. 762-3). This appliance has been slow to gain popularity in North America because of insufficient training and knowledge of the technique (Phan and Ling). On the basis of appearance, clear aligners and lingual braces are preferred over ceramic brackets, which are favored over stainless steel or hybrid appliances (Ziuchkovski et al.).

While fixed appliances are still very common today, the advent of removable clear aligners has undoubtedly revolutionized the orthodontic field in recent years. In 1945, Harold Kesling developed the Tooth Positioner as a finishing device to move teeth into more ideal occlusion. In 1971, Robert Ponitz began using thermoformed plastic for “invisible retainers" and for limited tooth movement. Displaced teeth were reset slightly on a dental model and a vacuum-formed sheet was made to fit the reset teeth, moving teeth into alignment. In the 1990s, John Sheridan popularized using these invisible "Essix" retainers with interproximal reduction (IPR) to move teeth (Graber et al. 778). Thus, the concept of an "aligner" was born.

Unlike with flexible archwires, only minor tooth movement is possible with a single clear aligner due to the stiffness of the thermoplastic material. One would need several models with teeth reset a small amount to fabricate a series of aligners for the patient to wear. To avoid the cost and complexity of making new aligners, Sheridan 
began using heated instruments to create "bumps" in the aligner, which effectively altered its shape and allowed re-use for minor changes (Graber et al. 778). However, a major drawback of this is that the plastic can only be stretched up to about $3 \mathrm{~mm}$ before it becomes too thin to exert enough force on a tooth. Since this process is both timeconsuming and imprecise, it is neither advised when moving more than a few teeth, nor when moving a single tooth more than $1 \mathrm{~mm}$ (Proffit et al. 354-5).

\section{B. Introduction of Invisalign}

In 1997, Align Technology developed Invisalign, which is arguably the most used and recognizable clear aligner system today. It was made available to clinicians in 1999 and aligns teeth using a series of customized computer-generated plastic aligners. These aligners are nearly invisible, as the name implies, and are meant to be worn 20 to 22 hours per day for one to two weeks, as prescribed by the clinician ("Living with Invisalign Clear Aligners").

To begin fabricating these clear aligners, a digital scan or polyvinyl siloxane (PVS) impression is taken of the patient's teeth. The scan or impression is sent to the company along with photographs and the clinician's treatment plan. A three-dimensional (3-D) digital model of the dentition is made and transmitted to a treatment facility where Invisalign technicians create a preliminary plan following the doctor's instructions. This 3-D interpretation of the plan is called a ClinCheck, which is available digitally for the doctor to review. Once any adjustments are made and the final plan approved by the doctor, stereolithographic (STL) models for each stage of alignment are 3-D printed. The aligners are formed from these models and sent directly to the doctor to deliver to the patient (Proffit et al. 355). 
When Invisalign was first introduced, the system was not received eagerly by orthodontists. Doubts arose regarding its effectiveness, as there were issues early on with staging of treatment, rates of tooth movement, and indications for attachment use (Proffit et al. 355). Today, these aligners can be used to treat dental malocclusions ranging from minor to severe. There are some movements that are performed well with clear aligner therapy, such as absolute intrusion of one to two teeth and closing mild to moderate spaces. Other movements, such as severe rotations of teeth with rounded roots and extrusion of incisors are difficult (Proffit et al. 356). To increase effectiveness, composite attachments are bonded to teeth so that the aligner can be more retentive and to facilitate tooth movement (Graber et al. 788).

There are currently two types of Invisalign attachments: optimized and conventional. Optimized attachments are engineered and patented by Align Technology to create precise biomechanical forces on teeth (Graber et al. 782). They are automatically placed by the ClinCheck software when a certain amount and type of planned tooth movement is detected. Conventional attachments are able to be chosen and manually placed by the doctor wherever deemed necessary (Graber et al. 789-90). The latter attachments are not unique to Invisalign and are used by other companies or with software to create in-office aligners with 3-D printers. Both attachment types come in various shapes, and some may be more useful than others for specific teeth and types of movement. While the precision of orthodontic tooth movements with Invisalign has been studied, the effectiveness of the different attachment types, amongst other aligner variables, has not been considered. 


\section{CHAPTER II: REVIEW OF LITERATURE}

\section{A. Invisalign Guidelines}

Since its introduction, Invisalign has recommended aligners to be worn for 22 hours a day to be effective. Originally, each Invisalign aligner was programmed to move a tooth 0.25 to $0.33 \mathrm{~mm}$ over fourteen days (Phan and Ling). In 2016, Invisalign changed its protocol from two-week wear to weekly aligner changes, decreasing treatment time by up to $50 \%$ (“The Invisalign Difference”).

In order to guide the clinician and help him or her assess planned tooth movements, Invisalign's ClinCheck website labels degree of movement by color. Align Technology divides movements into mild, moderate (blue), and advanced (black). Moderate movements indicate that mid-course correction, or "refinement" aligners, may be needed, along with IPR, attachments, buttons, or elastics. Advanced movements signify that refinement aligners and the adjuncts mentioned above will likely be necessary, possibly in addition to a first phase of treatment, sectional or fixed braces, or orthognathic surgery ("Invisalign Tooth Movement Assessment Overview"). Invisalign has altered its aligner material and has improved features over the years in an attempt to overcome these limitations and to increase predictability in movements.

\section{B. SmartTrack Material}

The first Invisalign aligners were made with a thermoplastic material called Proceed30 (PC30), which was a polymer mixture that did not meet all physical, chemical, and clinical requirements to effectively move teeth. In late 2001, Invisalign introduced a thinner polyurethane material called Exceed30 (EX30), which was four times more 
adaptable and 1.5 times more elastic than PC30, making removal and insertion easier. EX30 produced a greater force for orthodontic tooth movement, but clinicians reported that $70 \%$ to $80 \%$ of their cases needed mid-course correction with additional aligners (Condo et al).

Today, Align Technology uses SmartTrack (LD30), which is a multilayer polyurethane and copolyester material released in 2013 (Figure 1). Condo et al. found that SmartTrack was more flexible, delivered more gentle and constant forces, and was more adaptive to teeth than EX30. This study was conducted in vitro and the clinical effectiveness of SmartTrack material has yet to be examined. Other studies have shown that aligners have less impact on quality of life and that patients experience less pain than with traditional orthodontic braces (Graber et al. 785).

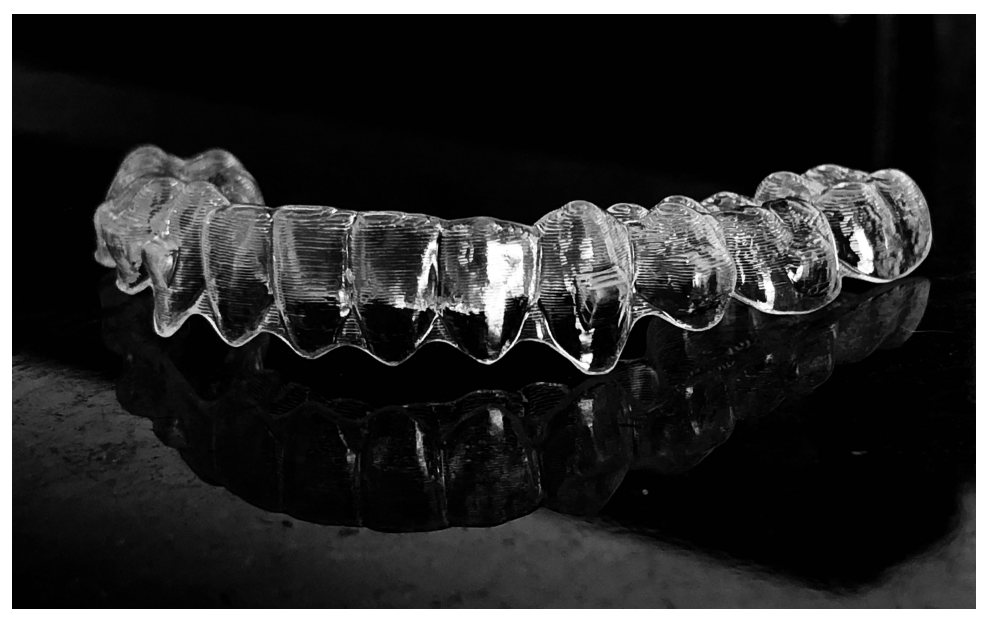

Figure 1. The Invisalign Appliance

\section{Invisalign Attachments}

The first Invisalign attachments were conventional attachments that were either ellipsoid in shape or rectangular horizontal or vertical (Figure 2). According to Graber et al., the ellipsoid shape is considered the least effective attachment today due to its small 
size and lack of a defined active surface. Before it became an option to bevel at attachment, which rotates a portion of it to be flush with the tooth surface, aligners were difficult to insert and remove (790). Attachment dimensions, prominence, degree of beveling, and position on the tooth may be changed according to clinician preference in the ClinCheck software (Align Technology Inc., "SmartForce Features"). Conventional attachments are still available and are widely used today.

In 2009, SmartForce features, which include optimized attachments, were developed. According to Align Technology, optimized attachments are designed to deliver optimal forces and moments based on individualized attachment shape and placement. As the aligner engages the attachment's active surface area, it is designed to simultaneously engage the lingual aspect of the tooth, resulting in a rotational movement down its long axis. Optimized rotation attachments are automatically placed onto canines or premolars when a rotation $\geq 5^{\circ}$ is detected (Figure 3). Maximum velocity is $2^{\circ}$ per aligner. Optimized extrusion attachments are applied onto incisors or canines when $\geq 0.5$ $\mathrm{mm}$ extrusion is detected by the software (Figure 4). Maximum velocity is $0.25 \mathrm{~mm}$ per aligner ("SmartForce Features"). One can see that optimized extrusion attachments are beveled to blend smoothly towards the gingival aspect of the tooth.

It is important to note that the attachment recess in the aligner is not the same shape as the optimized attachment. Unlike with conventional attachments, the software determines the shape of the aligner with two considerations: the "active" surface of the aligner engages and applies force onto the "active" surface of the attachment, while the "inactive" surface of the aligner has an intentional gap or reservoir, providing clearance for unimpeded tooth movement ("Tips for Success with Invisalign Optimized 
Attachments"). Although this may give the appearance that the attachment is not fully seated in the aligner, this excess space is intentional.

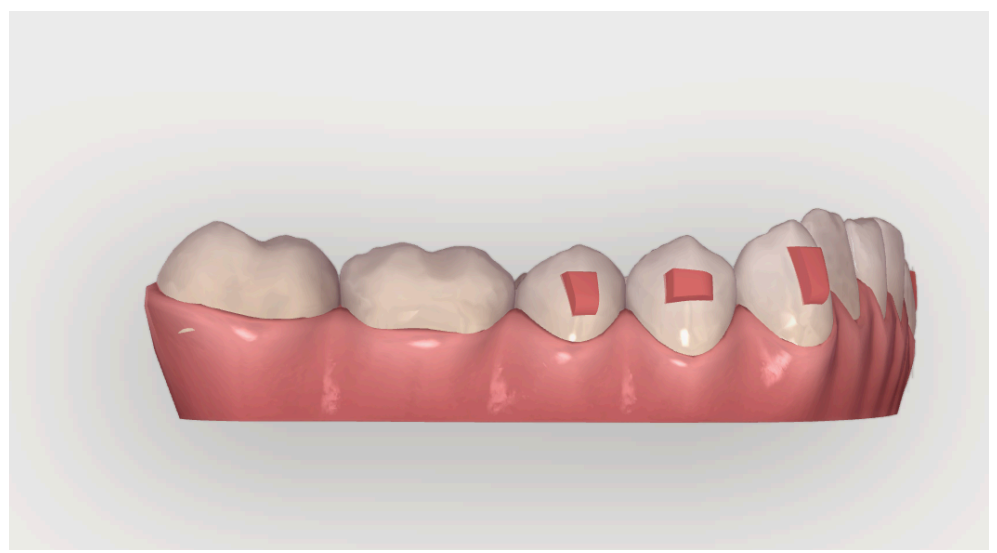

Figure 2. Conventional Rectangular Attachments

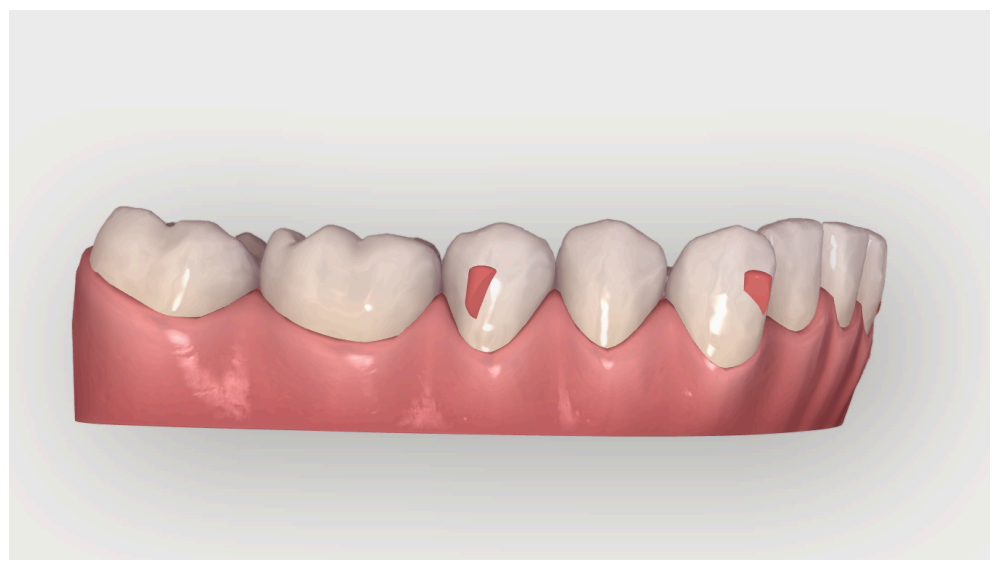

Figure 3. Optimized Rotation Attachments

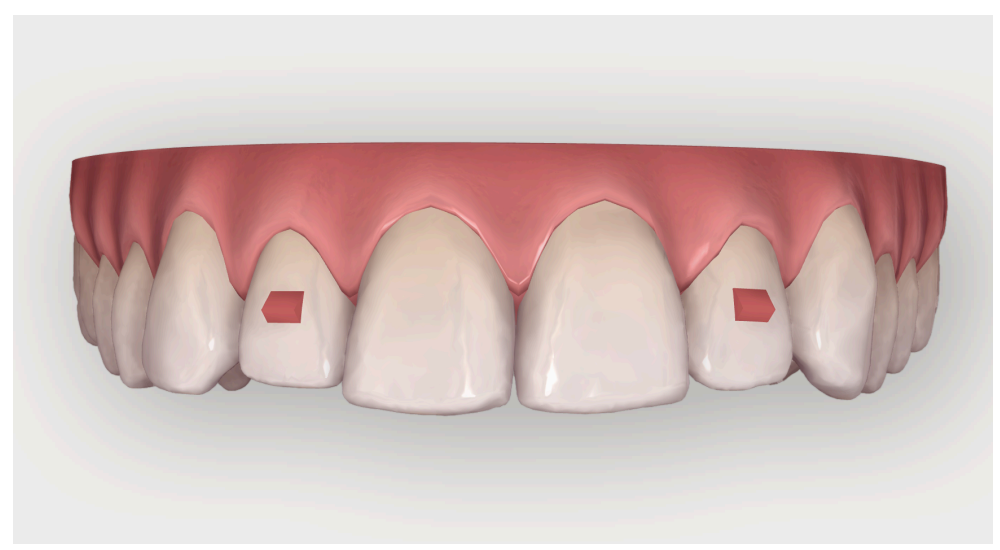

Figure 4. Optimized Extrusion Attachments 


\section{Accuracy of Invisalign}

One of the first studies to look at the accuracy of Invisalign was a prospective clinical trial published in 2009, a decade after the clear aligner system was introduced. Kravitz et al. superimposed predicted and achieved virtual models over unmoved posterior teeth using Invisalign's proprietary ToothMeasure software. The authors evaluated various types of tooth movement, including expansion, constriction, intrusion, extrusion, mesiodistal tip, labiolingual tip, and rotation. Using 37 patients and 401 anterior teeth, the overall mean accuracy of tooth movement was found to be $41 \%$. The most accurate movement was lingual constriction (47.1\%) and the least accurate was extrusion of incisors $(29.6 \%)$. For rotations, the least accurate tooth was the canine followed by the maxillary lateral incisor. Fifteen degrees was chosen as a clinically discernable amount of malrotation. For rotations $>15^{\circ}$, the accuracy of maxillary canines fell significantly (“How well does Invisalign work?”).

Shortly after, Kravitz et al. conducted another study examining the influence of [conventional] attachments and IPR on the accuracy of canine rotation, since this was the tooth found to be the least accurate for that movement. The same superimposition software was used and compared movements of teeth with attachments only, IPR only, and neither attachments nor IPR. The mean accuracy of canine rotation was $35.8 \%$. Canines that received IPR had the highest mean accuracy and presence of attachments only modestly improved accuracy. However, vertical-ellipsoid attachments (the most commonly prescribed attachment) and IPR did not significantly improve the accuracy of canine rotation (“Influence of attachments and interproximal reduction”). 
Limitations of these two studies include the fact that location of the attachments was not accounted for, though recommendations from Align Technology Inc. at the time included placement at the incisal third of the tooth to maximize aligner retention (Kravitz et al., "Influence of attachments and interproximal reduction"). Optimized attachments were not yet available and ellipsoid attachments are rarely used today. Additionally, the sample sizes were small, and the periodontal anchorage of stationary posterior teeth used for superimpositions was not considered.

To eliminate the limitation of the potential movement of reference teeth, Krieger et al. analyzed the accuracy of Invisalign in the anterior tooth region by measuring intermaxillary rather than intramaxillary measurements. Overbite, overjet, and midline shift in patients with mild to moderate crowding was again assessed using Invisalign's ToothMeasure tool, which is no longer available for doctor use. In their pilot study with 35 patients, it was found that movements in the vertical plane (overbite) were the most difficult to achieve, with a $0.9 \mathrm{~mm}$ mean discrepancy between predicted and achieved measurements (“Accuracy of Invisalign treatments"). When a follow-up study was conducted with a larger sample size of 50 patients and more parameters, it was again found that overbite displayed the greatest deviations between predicted and achieved measurements by a mean of $0.7 \mathrm{~mm}$ discrepancy ("Invisalign treatment in the anterior region"). The authors suggested vertical overcorrection in the final ClinCheck, refinement aligners or adjuncts (i.e. supportive attachments on adjacent teeth or vertical elastics) to improve predictability.

In 2014, Simon et al. further investigated the accuracy of Invisalign using 30 patients in a retrospective split-mouth study. They found that the mean accuracy was 
$40 \%$ for premolar derotation $>10^{\circ}, 42 \%$ for upper incisor torque $>10^{\circ}$, and $87 \%$ for molar distalization $>1.5 \mathrm{~mm}$. The mean accuracy for premolar derotation was $42.4 \%$ without and $47.3 \%$ with the support of an attachment when eliminating cases of poor patient compliance. The results also showed that the accuracy was significantly reduced for predicted rotations $>15^{\circ}$ with a mean accuracy of $23.6 \%$. For premolar derotations with staging $<1.5^{\circ}$ per aligner, the total efficacy was $41.8 \%$, while the accuracy decreased to $23.2 \%$. with staging $>1.5^{\circ}$ per aligner. Therefore, premolar derotation significantly depends on velocity and total amount of planned tooth movement. ("Treatment outcome and efficacy of an aligner technique").

A second experimental study by Simon et al. examined forces and moments generated by aligners. It was found that for the premolar derotation group, the measured moments differed significantly depending when an attachment was used. The findings indicated that load transfer from an aligner to a cylindrical tooth without an attachment is possible only to a limited extent. Their results corresponded with results in the literature stating that derotation of cylindrical teeth is one of the most difficult movements to be achieved with clear aligners. ("Forces and moments generated by removable thermoplastic aligners").

In 2018, Charalampakis et al. conducted a retrospective study examining the accuracy of Invisalign using the 3D Slicer CMF software. All orthodontic cases considered for this study were begun after 2014, which is when Invisalign's SmartTrack clear aligner material was introduced. Predicted and achieved models from 20 patients were superimposed over the initial ones to compare the differences between predicted and achieved movements. They found statistically significant inaccuracies for intrusion 
of incisors and for rotations of all teeth, especially canines, which had a mean discrepancy of $3.05^{\circ}$ in the maxillary arch and $2.45^{\circ}$ in the mandibular arch. Maxillary premolars had the lowest discrepancy of only $0.9^{\circ}$. Unlike in earlier studies, interpremolar expansion, along with horizontal movement and extrusion of incisors, were found to be accurate. However, like previous studies, sample size was small, and the potential movement of the "stationary" teeth superimposed on was not considered, which may affect vertical measurements the most.

Although the aforementioned studies are valuable in determining the accuracy and predictability of the Invisalign system itself, none of them compared final outcomes to clinically acceptable standards of care. Buschang et al. used the American Board of Orthodontics Objective Grading System (ABO OGS) to assess 27 orthodontic cases treated with Invisalign. The final predicted models displayed in the ClinCheck showed significantly fewer point deductions compared to the final achieved models, suggesting that the predicted models do not accurately reflect the patients' final occlusion. Differences were mainly observed in alignment, buccolingual inclinations, occlusal contacts, and occlusal relations. However, even after these point deductions, the aligner cases were still found to be clinically acceptable.

In 2018, a systematic review assessing the available research on the clinical effectiveness of Invisalign was published. The overall level of evidence of the included studies was moderate, and the risk of bias ranged from low to high. It concluded that Invisalign can predictably level, tip, and derotate anterior teeth, but not canines and premolars. Limitations of Invisalign also include posterior arch expansion through bodily tooth movement, closure of extraction spaces, improvement of occlusal contacts, 
extrusion of maxillary incisors, and correction of large antero-posterior and vertical discrepancies (Papadimitriou et al.).

\section{E. Invisalign vs. Fixed Appliances}

The same systematic review also compared Invisalign treatment outcomes to that of conventional fixed appliances. In regard to treatment time, Invisalign was shown to be significantly faster than fixed appliances in three studies, while no difference was reported in another study. However, when extractions were considered, Invisalign treatment duration was longer. Thus, it seems that Invisalign might treat mild nonextraction cases faster than fixed appliances but may require more time for complex cases (Papadimitriou et al.).

Though Invisalign treatment time may be less than with fixed appliances, it does not mean it is as effective. Three studies used the ABO grading system to score pre- and post-treatment models and found that treatment results of fixed appliances were superior to those of Invisalign. However, both treatment modalities fulfilled all ABO objectives. One study also found greater relapse in patients treated with Invisalign over a posttreatment observation period of one to three years. This may be due to inadequacies in attaining bodily movement and stable occlusal contacts (Papadimitriou et al.). Overall, no clear clinical recommendations could be made, besides that Invisalign is a viable alternative to conventional fixed appliances for the correction of mild to moderate malocclusions in adult patients that do not require extraction (Papadimitriou et al.). 


\section{CHAPTER III: OBJECTIVE AND HYPOTHESES}

The aim of this research was to compare the efficacy of optimized and conventional attachment types on rotations of cylindrical teeth (canines and premolars) and on extrusion of anterior teeth - two movements reported to be the most difficult to achieve predictably with Invisalign. Results may have a clinical impact, as they can help guide dentists in their choice of attachment types when treatment planning with Invisalign or another clear aligner software.

The null hypotheses were: 1) there will be no statistically significant difference between the accuracy of optimized and conventional attachments for rotation of canines and premolars, and 2) there will be no statistically significant difference between the accuracy of optimized and conventional attachments for extrusion of incisors and canines. 


\section{CHAPTER IV: MATERIALS AND METHODS}

\section{A. Sample Description}

This retrospective study consisted of 382 teeth from digital dental models of 100 orthodontic patients ranging from ages 11 to 63 years (32 males and 68 females with a mean age of 28 years, 2 months) (Figure 5). All patients were treated with Invisalign (Align Technology, Santa Clara, CA) by one of two orthodontists in private practice outside of Milwaukee, WI and Chicago, IL between January 2016 and August 2018. One of the orthodontists had been providing Invisalign since 2011 and the other since 2013. Aligners were changed according to both the manufacturer's and clinician's recommendations at the time, which was either every two weeks or once a week. The study protocol was approved by the Institutional Review Board (IRB) of Marquette University (HR-1811026964).

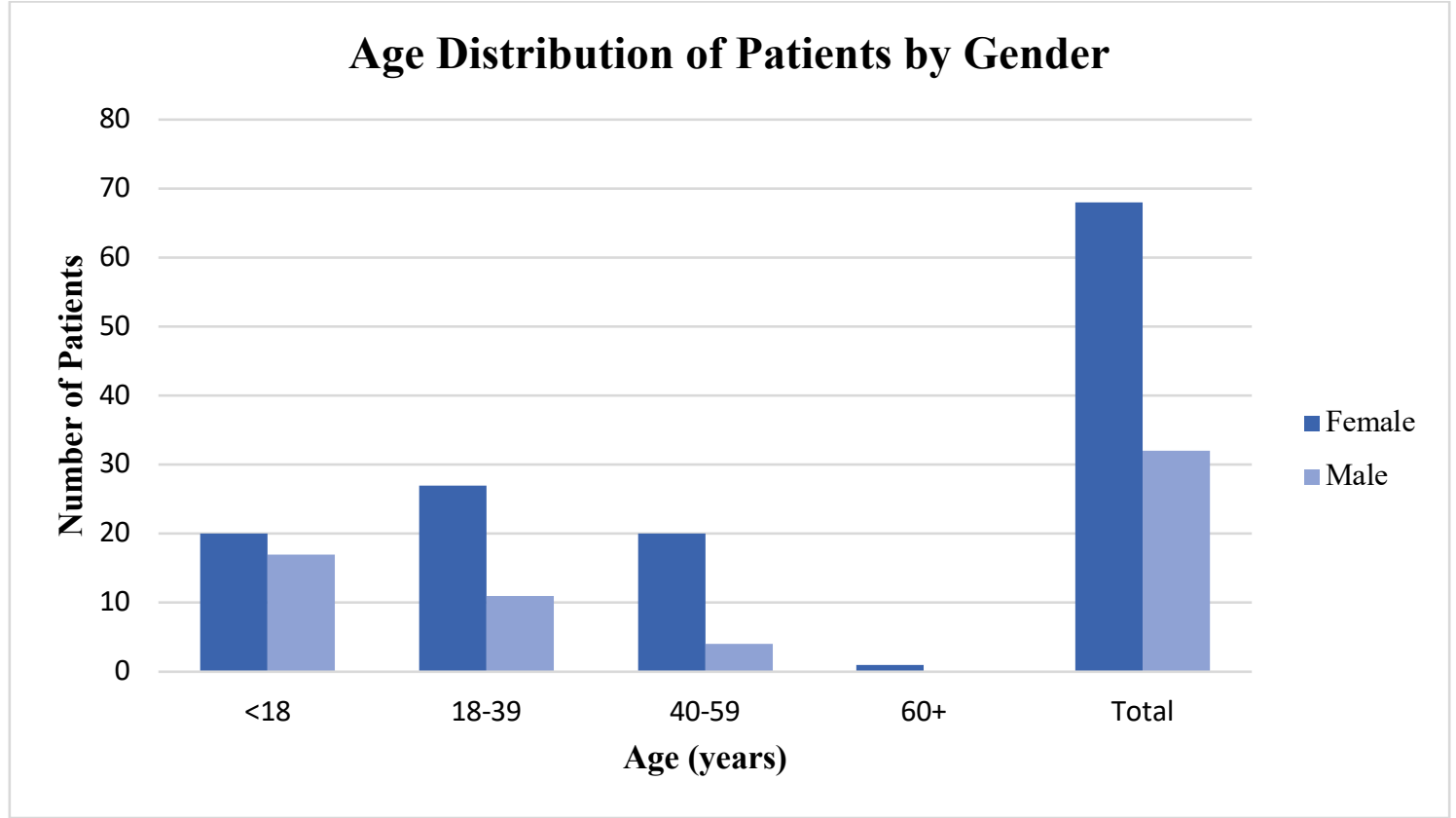

Figure 5. Patient Characteristics 
A power analysis indicated that a sample size of 64 teeth per group would be needed to have a power of $95 \%$ with a type I error of $P=0.05$. The number of attachment types were: 163 optimized rotation (43\%), 72 conventional rotation (19\%), 81 optimized extrusion (21\%), and 66 conventional extrusion (17\%). The average number of aligners per series was 20. Spacing was present or IPR performed on either side of 61 out of the 382 teeth being studied (16\%).

To achieve a higher sample size for conventional attachment types, 15 of the 100 patients were used twice, 1 was used three times, and 1 was used four times. These patients were considered separate subjects for data collection purposes by using a refinement scan as a new "initial" model, and either the following refinement scan or the final scan at the end of treatment as the "achieved" model (whichever came first).

\section{B. Eligibility Criteria}

Main inclusion criteria were: (1) the presence of optimized or conventional rotation or extrusion attachments in the planned ClinCheck (Align Technology, Santa Clara, CA); (2) completion of the initial series of aligners, resulting in either a refinement or final scan; (3) no planned movement of at least one posterior tooth per side of the dental arch (for superimposition purposes); (4) reported good compliance with aligner wear; (5) full permanent dentition; and (6) treatment beginning in 2014 or later, after the release of the SmartTrack material (Align Technology, Santa Clara, CA).

The exclusion criteria were: (1) patients in the primary or mixed dentition; (2) new dental restorations or extractions during treatment; (3) the use of any auxiliaries, such as elastics or vibrational devices; and (4) patients with any orofacial syndromes or malformations. 


\section{Data Collection}

The principal investigator visited the private practices ten times throughout the experimental period to collect data. The following information was collected from the Invisalign patient charts and the office charts: patient identification number, gender, age at the start of treatment, dates of initial scans and refinement or final scans, number of trays per series of aligners, attachment types on individual teeth, amount and location of spacing or IPR, and patient compliance. The data were recorded in a master spreadsheet on the researcher's personal computer and saved on an encrypted USB flash drive. The USB drive was securely stored in the Orthodontic Department at the Marquette University School of Dentistry. After going through the orthodontists' accounts on the Invisalign doctor website, 128 potential subjects were identified. Upon reviewing the offices' digital charts for compliance and other factors, 100 met the inclusion and exclusion criteria.

In order to detect which teeth had conventional attachments placed primarily for rotation or extrusion, the previous unaccepted ClinChecks were reviewed to confirm that an optimized rotation or extrusion attachment was removed and then replaced by a conventional one. Removal and replacement of an optimized attachment would indicate that conventional attachments were placed on teeth with planned rotations $\geq 5^{\circ}$ or extrusion $\geq 0.5 \mathrm{~mm}$, which are the thresholds for optimized attachments to be placed.

\section{Model Superimposition}

Initial, predicted, and achieved digital dental models were exported from the ClinCheck software as stereolithography (STL) files. The initial and final models from 
the original ClinCheck were labeled as "initial" and "predicted," respectively. The models from the mid-treatment refinement scan or the models from the final scan at the end of treatment (whichever came first) were labeled as "achieved." These STL files were then imported into the 3D Slicer CMF program (open source, version 4.7.0) for superimpositions and measurements.

In order to superimpose the models, fiducial markers were placed in the central pit of the occlusal surfaces of posterior teeth planned to have no movement. These were most often second molars, but sometimes included first molars and premolars. From these fiducials, a region of interest (ROI) was created by increasing the value of the radius to include the entire occlusal surface, at a minimum. Using the ROIs, initial and predicted models were superimposed onto each other to measure "predicted" tooth movements, while initial and achieved models were superimposed to measure "achieved" movements (Figure 6).
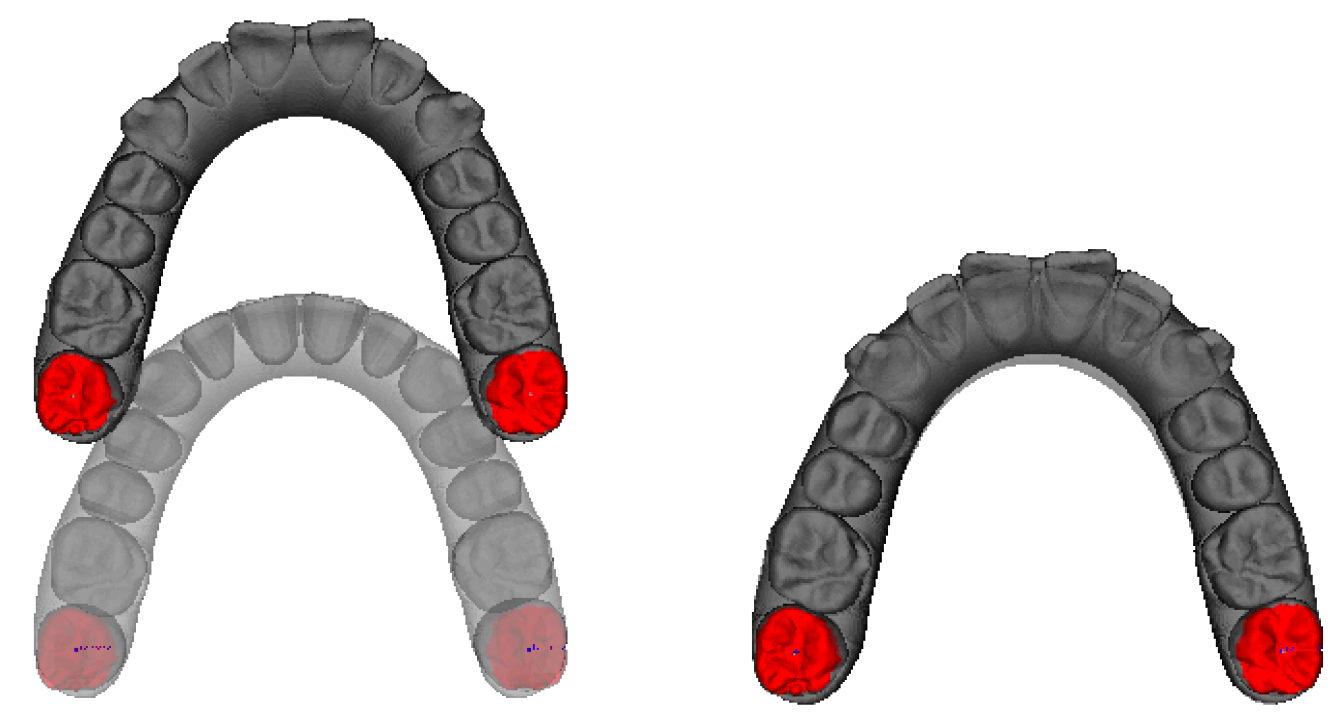

Figure 6. 3-D Model Superimposition Using 3D Slicer CMF On the left, regions of interest (ROIs) on unmoved second molars of initial (top) and predicted (bottom) maxillary arches. On the right, arches after they were superimposed. 


\section{E. Measurements}

Measurements were made on 382 teeth as follows: (1) Rotations of canines and premolars - two landmarks were manually placed on each tooth, the points were automatically connected to form a straight line, and the angle (yaw), between the two lines was calculated by the software in degrees $\left(^{\circ}\right)$ (Figure 7). The landmarks used were usually buccal and lingual cusp tips on premolars or a cusp tip and cingulum on canines. If the cusp tips or cingula were ill-defined or the points not reproducible, the most mesial and distal points of each tooth were used. (2) Extrusion of incisors and canines - one point was chosen near the center of the incisal edge or cusp tip of each tooth, and the distance between the two points was calculated in millimeters (mm) (Figure 8).

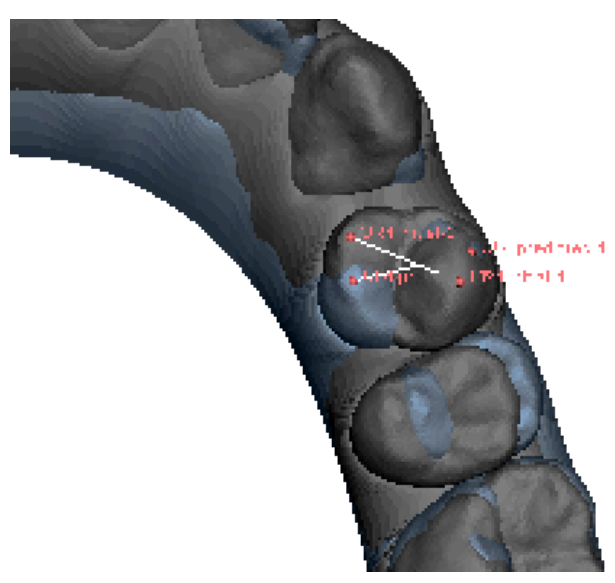

Figure 7. Rotational Measurements

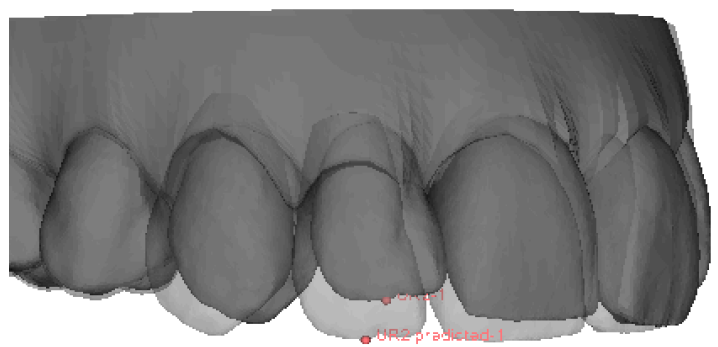

Figure 8. Extrusive Measurements

To account for any error in model superimposition due to inadvertent vertical movement of "stationary" posterior teeth, all "achieved" extrusive measurements were adjusted by comparing them to a control tooth. The control teeth were usually directly adjacent to those being measured so that they were roughly in the same antero-posterior (A-P) position along the dental arch. This is because posterior intrusion would affect 
teeth differently depending on their A-P location. In addition to being near teeth with planned extrusion, control teeth were measured to confirm no predicted vertical movement $(0 \pm 0.05 \mathrm{~mm})$. Achieved movement of these control teeth was measured to be able to calculate the true extrusion that resulted for the adjacent teeth in interest.

For example, if a maxillary lateral incisor had planned extrusion of $0.5 \mathrm{~mm}$ and $0.8 \mathrm{~mm}$ was measured to be achieved, then the adjacent maxillary canine that had no planned vertical movement was used as the control to adjust for any error in superimposition. If the maxillary canine was found to extrude $0.6 \mathrm{~mm}$, it was assumed this was due to posterior intrusion from plastic thickness. To calculate the true extrusion of the maxillary lateral incisor, $0.6 \mathrm{~mm}$ (the achieved movement of the canine) was subtracted from $0.8 \mathrm{~mm}$ (the perceived extrusion of the lateral incisor) for a total true extrusion of $0.2 \mathrm{~mm}$. All "achieved" extrusive measurements were adjusted in this manner.

\section{F. Statistical Analysis}

To calibrate the principal investigator to a uniform measuring method, all of the measurements were performed only after initially completing several measurements as a practice exercise. The same examiner repeated 40 of the rotational measurements and 40 of the extrusive measurements by random within a 3-week interval to assess intraexaminer reliability by means of an intraclass correlation coefficient (ICC). Data analysis was performed using Statistical Analysis Software (SAS version 9.4, SAS Institute, Cary, NC).

Any tooth that was measured to have a small negative achieved value for extrusion (indicating intrusion) was changed to $0 \mathrm{~mm}$ since no extrusion was achieved. 
This was done to avoid large negative percentages when calculating accuracy (percent accuracy $=100 \%-[\mid$ predicted - achieved $|/|$ predicted $\mid \times 100 \%])$. In this equation, the absolute value of the difference between predicted and achieved movements was taken to ensure that percent accuracy never exceeded $100 \%$ for the teeth that achieved movements beyond what was predicted. To account for this same situation, the absolute value was also taken when calculating the discrepancy between predicted and achieved measurements in degrees and millimeters to avoid yielding negative values that would affect the mean without accounting for directionality.

To reduce the number of variables, similar types of teeth were grouped together, including contralateral teeth, maxillary first and second premolars, mandibular first and second premolars, and mandibular central and lateral incisors. Descriptive statistics were calculated for both tooth movements and attachment types. Paired $t$-tests were used to compare mean predicted and mean achieved movements within groups. Independent $t$ tests were used to compare mean predicted and mean achieved movements between optimized and conventional attachments. One-way ANOVAs were used to compare mean accuracies of movements amongst tooth groups. Data analysis was performed at the $P<0.05$ significance level. 


\section{CHAPTER V: RESULTS}

\section{A. Intra-examiner Reliability}

The intraclass correlation coefficient (ICC) was excellent with a score of 0.970 [95\% CI 0.944, 0.984] for overall mean difference values. For rotation, Cronbach's alpha was 0.965 [ $95 \%$ CI $0.914,0.986]$. For extrusion, intra-rater reliability had a value of $0.907[95 \%$ CI $0.780,0.962]$.

\section{B. Sample Characteristics}

While 100 patients met all of the eligibility criteria, 17 of those patients were used more than once for a total of 120 subjects. The sample size consisted of 97 maxillary arches, 60 mandibular arches, and 382 teeth. The most common conventional attachment shape for rotations was rectangular vertical, while for extrusion it was rectangular horizontal with a gingival bevel. Both optimized and conventional rotation attachments were most commonly found on mandibular premolars ( $\mathrm{n}=54$ and $\mathrm{n}=26$, respectively), while optimized and conventional extrusion attachments were most frequently placed on maxillary lateral incisors ( $\mathrm{n}=40$ and $\mathrm{n}=30$, respectively). The most frequently evaluated tooth in general was the maxillary canine $(n=87)$. This is because canines were the only teeth studied that could have either a rotation or extrusion attachment. The tooth category with the least measurements was the mandibular incisor $(\mathrm{n}=16)$.

The amount of predicted tooth movement was divided into groups according to Align Technology's classifications: extrusion was divided into mild $(<2.5 \mathrm{~mm})$, moderate (2.5-3.5 mm), or advanced $(>3.5 \mathrm{~mm})$, while rotation was also organized into mild $\left(<45^{\circ}\right)$, moderate $\left(45-55^{\circ}\right)$, or advanced $\left(>55^{\circ}\right)$ ("Invisalign Tooth Movement Assessment 
Overview"). As seen in Figures 9 and 10, the majority of predicted tooth movements were mild rotations $<45^{\circ}$ and mild extrusion $<2.5 \mathrm{~mm}$. The sample sizes for moderate and advanced movements were too small to perform valuable analyses between these three ranges. Predicted rotations ranged from $2.19^{\circ}$ to $74.00^{\circ}$, while planned extrusion ranged from $0.18 \mathrm{~mm}$ to $4.21 \mathrm{~mm}$.

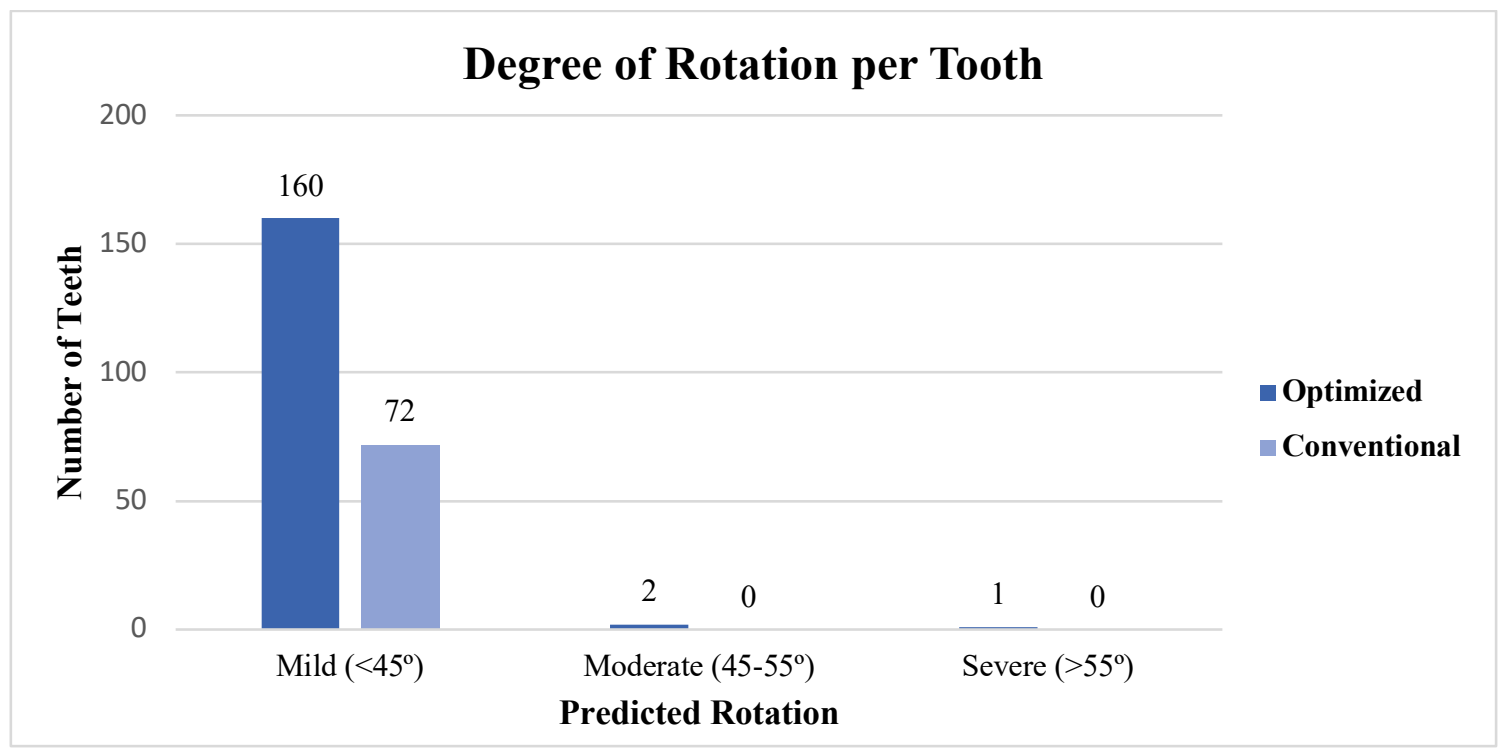

Figure 9. Tooth Movement Characteristics: Predicted Rotation

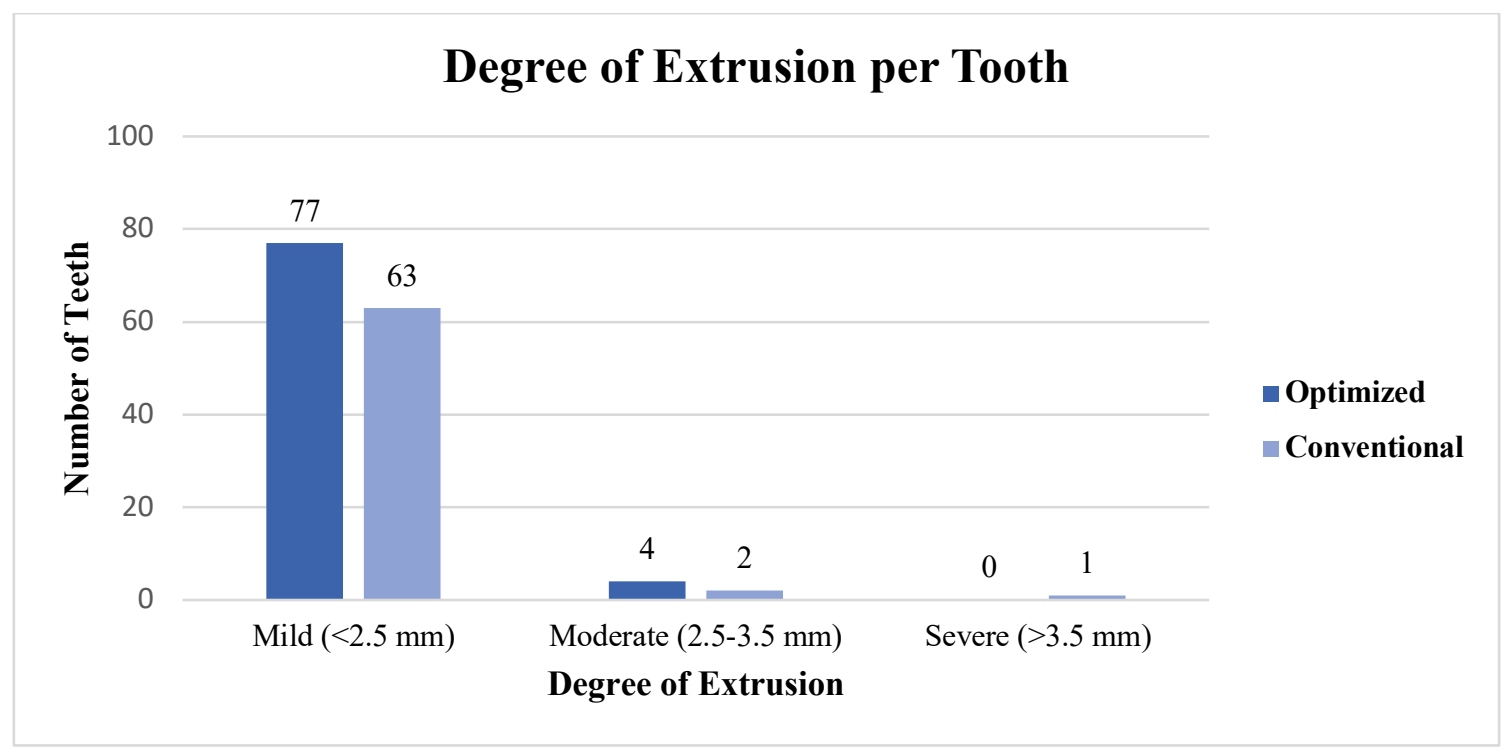

Figure 10. Tooth Movement Characteristics: Predicted Extrusion 


\section{Predicted vs. Achieved Movements}

Descriptive statistics for predicted and achieved values for both optimized and conventional attachment types are presented in Tables 1-4. Absolute mean difference, which would ideally be zero, was computed by subtracting the achieved amount of movement from the predicted amount for each tooth and then taking the absolute value. Absolute values were taken to avoid a negative difference, which occurred in a few cases where achieved values were higher than predicted measurements.

For the entire sample, average predicted rotation was $14.05^{\circ}$, while average achieved rotation was $9.43^{\circ}$. Average predicted extrusion was $1.11 \mathrm{~mm}$ and average achieved extrusion was $0.61 \mathrm{~mm}$.

Table 1. Descriptive Statistics for Optimized Rotation Attachments

\begin{tabular}{|c|c|c|c|c|}
\hline Tooth & Movement & $\mathbf{n}$ & Mean $\left({ }^{\circ}\right)$ & SD \\
\hline \multirow{3}{*}{$\begin{array}{l}\text { Maxillary } \\
\text { canine }\end{array}$} & Predicted & \multirow{3}{*}{38} & 14.26 & 9.97 \\
\hline & Achieved & & 9.71 & 7.37 \\
\hline & |Predicted - Achieved $\mid$ & & 4.94 & 6.62 \\
\hline \multirow{3}{*}{$\begin{array}{l}\text { Maxillary } \\
\text { premolar }\end{array}$} & Predicted & \multirow{3}{*}{36} & 12.65 & 12.73 \\
\hline & Achieved & & 9.68 & 8.83 \\
\hline & |Predicted - Achieved| & & 3.54 & 5.86 \\
\hline \multirow{3}{*}{$\begin{array}{l}\text { Mandibular } \\
\text { canine }\end{array}$} & Predicted & \multirow{3}{*}{35} & 15.49 & 11.04 \\
\hline & Achieved & & 12.23 & 9.61 \\
\hline & |Predicted - Achieved $\mid$ & & 3.89 & 4.60 \\
\hline \multirow{3}{*}{$\begin{array}{l}\text { Mandibular } \\
\text { premolar }\end{array}$} & Predicted & \multirow{3}{*}{54} & 14.42 & 8.49 \\
\hline & Achieved & & 9.62 & 7.95 \\
\hline & |Predicted-Achieved| & & 5.74 & 5.59 \\
\hline \multirow{3}{*}{ Total } & Predicted & \multirow{3}{*}{163} & 14.22 & 10.38 \\
\hline & Achieved & & 10.21 & 8.39 \\
\hline & |Predicted - Achieved| & & 4.67 & 5.73 \\
\hline
\end{tabular}


Table 2. Descriptive Statistics for Conventional Rotation Attachments

\begin{tabular}{|c|c|c|c|c|}
\hline Tooth & Movement & $\mathbf{n}$ & Mean $\left({ }^{\circ}\right)$ & SD \\
\hline \multirow{3}{*}{$\begin{array}{l}\text { Maxillary } \\
\text { canine }\end{array}$} & Predicted & \multirow{3}{*}{17} & 11.18 & 7.29 \\
\hline & Achieved & & 7.11 & 6.61 \\
\hline & |Predicted - Achieved| & & 4.45 & 4.59 \\
\hline \multirow{3}{*}{$\begin{array}{l}\text { Maxillary } \\
\text { premolar }\end{array}$} & Predicted & \multirow{3}{*}{10} & 11.94 & 8.79 \\
\hline & Achieved & & 5.08 & 2.83 \\
\hline & |Predicted-Achieved| & & 6.86 & 7.73 \\
\hline \multirow{3}{*}{$\begin{array}{l}\text { Mandibular } \\
\text { canine }\end{array}$} & Predicted & \multirow{3}{*}{19} & 15.78 & 8.53 \\
\hline & Achieved & & 9.50 & 7.71 \\
\hline & |Predicted-Achieved| & & 6.84 & 6.39 \\
\hline \multirow{3}{*}{$\begin{array}{c}\text { Mandibular } \\
\text { premolar }\end{array}$} & Predicted & \multirow{3}{*}{26} & 14.41 & 8.93 \\
\hline & Achieved & & 7.66 & 4.80 \\
\hline & |Predicted-Achieved $\mid$ & & 6.84 & 8.23 \\
\hline \multirow{3}{*}{ Total } & Predicted & \multirow{3}{*}{72} & 13.67 & 8.46 \\
\hline & Achieved & & 7.66 & 6.00 \\
\hline & |Predicted-Achieved $\mid$ & & 6.28 & 6.90 \\
\hline
\end{tabular}

Table 3. Descriptive Statistics for Optimized Extrusion Attachments

\begin{tabular}{|c|c|c|c|c|}
\hline Tooth & Movement & $\mathbf{n}$ & Mean (mm) & SD \\
\hline \multirow{3}{*}{$\begin{array}{l}\text { Maxillary central } \\
\text { incisor }\end{array}$} & Predicted & \multirow{3}{*}{11} & 1.45 & 0.77 \\
\hline & Achieved & & 1.36 & 1.25 \\
\hline & |Predicted-Achieved & & 0.52 & 0.37 \\
\hline \multirow{3}{*}{$\begin{array}{l}\text { Maxillary lateral } \\
\text { incisor }\end{array}$} & Predicted & \multirow{3}{*}{40} & 1.00 & 0.51 \\
\hline & Achieved & & 0.54 & 0.49 \\
\hline & |Predicted-Achieved & & 0.50 & 0.31 \\
\hline \multirow{3}{*}{ Maxillary canine } & Predicted & \multirow{3}{*}{19} & 1.01 & 0.85 \\
\hline & Achieved & & 0.52 & 0.48 \\
\hline & |Predicted-Achieved| & & 0.50 & 0.53 \\
\hline \multirow{3}{*}{$\begin{array}{l}\text { Mandibular } \\
\text { incisor }\end{array}$} & Predicted & \multirow{3}{*}{9} & 1.10 & 0.87 \\
\hline & Achieved & & 0.72 & 0.54 \\
\hline & |Predicted-Achieved & & 0.38 & 0.42 \\
\hline \multirow{3}{*}{$\begin{array}{l}\text { Mandibular } \\
\text { canine }\end{array}$} & Predicted & \multirow{3}{*}{2} & 0.59 & 0.37 \\
\hline & Achieved & & 0.23 & 0.12 \\
\hline & |Predicted-Achieved| & & 0.37 & 0.49 \\
\hline \multirow{3}{*}{ Total } & Predicted & \multirow{3}{*}{81} & 1.06 & 0.68 \\
\hline & Achieved & & 0.66 & 0.69 \\
\hline & Predicted - Achieved & & 0.49 & 0.38 \\
\hline
\end{tabular}


Table 4. Descriptive Statistics for Conventional Extrusion Attachments

\begin{tabular}{|c|c|c|c|c|}
\hline Tooth & Movement & $\mathbf{n}$ & Mean (mm) & SD \\
\hline \multirow{3}{*}{$\begin{array}{l}\text { Maxillary central } \\
\text { incisor }\end{array}$} & Predicted & \multirow{3}{*}{11} & 1.37 & 1.21 \\
\hline & Achieved & & 0.94 & 0.73 \\
\hline & |Predicted - Achieved| & & 0.44 & 0.53 \\
\hline \multirow{3}{*}{$\begin{array}{l}\text { Maxillary lateral } \\
\text { incisor }\end{array}$} & Predicted & \multirow{3}{*}{30} & 1.03 & 0.58 \\
\hline & Achieved & & 0.51 & 0.37 \\
\hline & |Predicted - Achieved $\mid$ & & 0.52 & 0.37 \\
\hline \multirow{3}{*}{ Maxillary canine } & Predicted & \multirow{3}{*}{13} & 1.23 & 0.75 \\
\hline & Achieved & & 0.45 & 0.43 \\
\hline & |Predicted - Achieved| & & 0.78 & 0.57 \\
\hline \multirow{3}{*}{$\begin{array}{l}\text { Mandibular } \\
\text { incisor }\end{array}$} & Predicted & \multirow{3}{*}{7} & 1.46 & 0.67 \\
\hline & Achieved & & 0.55 & 0.66 \\
\hline & |Predicted-Achieved| & & 0.92 & 0.29 \\
\hline \multirow{3}{*}{$\begin{array}{l}\text { Mandibular } \\
\text { canine }\end{array}$} & Predicted & \multirow{3}{*}{5} & 1.00 & 0.47 \\
\hline & Achieved & & 0.22 & 0.28 \\
\hline & |Predicted-Achieved| & & 0.77 & 0.30 \\
\hline \multirow{3}{*}{ Total } & Predicted & \multirow{3}{*}{66} & 1.17 & 0.75 \\
\hline & Achieved & & 0.55 & 0.51 \\
\hline & |Predicted-Achieved| & & 0.62 & 0.45 \\
\hline
\end{tabular}

Paired $t$-tests were used to compare mean predicted and achieved values for each of the four groups studied (Table 5). For all tooth movements and attachment types, mean predicted values were larger than mean achieved values at $P<0.0001$, indicating high statistical significance. Mean differences were higher for conventional attachments.

Table 5. Predicted Movements vs. Achieved Movements

\begin{tabular}{|c|c|c|c|c|c|c|}
\hline \multirow{2}{*}{$\begin{array}{c}\text { Attachment and } \\
\text { Tooth Movement }\end{array}$} & $\mathbf{n}$ & $\begin{array}{c}\text { Mean Difference } \\
\text { (Predicted - Achieved) }\end{array}$ & SD & \multicolumn{2}{|c|}{$\mathbf{9 5 \%}$ CI } & \multirow{2}{*}{ P-value } \\
\hline $\begin{array}{c}\text { Optimized } \\
\text { Rotation (') * }\end{array}$ & 163 & 4.01 & 6.22 & 3.05 & 4.97 & $<0.0001$ \\
\hline $\begin{array}{c}\text { Conventional } \\
\text { Rotation (') }\end{array}$ & 72 & 6.01 & 7.14 & 4.33 & 7.68 & $<0.0001$ \\
\hline $\begin{array}{c}\text { Optimized } \\
\text { Extrusion (mm) * }\end{array}$ & 81 & 0.40 & 0.47 & 0.30 & 0.51 & $<0.0001$ \\
\hline $\begin{array}{c}\text { Conventional } \\
\text { Extrusion (mm) * }\end{array}$ & 66 & 0.62 & 0.45 & 0.51 & 0.73 & $<0.0001$ \\
\hline
\end{tabular}

$* P<0.05$ 
Two separate one-way ANOVAs were performed in order to compare mean differences between tooth types for both rotation and extrusion (Table 6). There was no statistically significant difference between tooth types for rotation $(P>0.05)$ and for extrusion $(P>0.05)$. Therefore, no post-hoc analyses were needed.

Table 6. ANOVA Comparing Absolute Mean Difference by Tooth Type

\begin{tabular}{|c|c|c|c|c|}
\hline Tooth Movement & df & Mean Square & F & $P$-value \\
\hline Rotation $\left(\mathbf{(}^{\mathbf{}}\right)$ & 3 & 39.24 & 1.04 & 0.3757 \\
\hline Extrusion $(\mathbf{m m})$ & 4 & 0.13 & 0.70 & 0.5898 \\
\hline
\end{tabular}

\section{Mean Accuracy}

Mean accuracy for all tooth groups was calculated for a better understanding of the Invisalign system's precision (percent accuracy $=100 \%$ - [|predicted - achieved $\mid /$ |predicted| x 100\%]) (Table 7). Sixteen teeth were shown to intrude an average of 0.16 $\mathrm{mm}$, so these achieved movements were changed to $0 \mathrm{~mm}$ for extrusion, yielding a $0 \%$ accuracy.

Mean accuracy for all teeth studied was 57.2\%. Mean accuracy for rotation was $63.2 \%$, while for extrusion it was $47.6 \%$. The most severe planned movements for both rotation $\left(74.0^{\circ}\right)$ and extrusion $(4.21 \mathrm{~mm})$ had an accuracy of $64 \%$. The least accurate tooth movement was mandibular canine extrusion with a conventional attachment (16.1\%). The most accurate tooth movement was extrusion of the maxillary central incisor with a conventional attachment (73.9\%), followed closely by rotation of the maxillary premolar with an optimized attachment $(72.8 \%)$. 
Table 7. Descriptive Statistics for Mean Accuracy

\begin{tabular}{|c|c|c|c|c|}
\hline Tooth & $\begin{array}{c}\text { Attachment and } \\
\text { Tooth Movement }\end{array}$ & $\mathbf{n}$ & Mean Accuracy (\%) & SD \\
\hline \multirow{2}{*}{$\begin{array}{c}\text { Maxillary } \\
\text { central incisor }\end{array}$} & Conventional extrusion & 11 & 73.9 & 18.4 \\
\cline { 2 - 5 } $\begin{array}{c}\text { Maxillary } \\
\text { lateral incisor }\end{array}$ & Optimized extrusion & 11 & 58.7 & 24.6 \\
\hline \multirow{4}{*}{$\begin{array}{c}\text { Maxillary } \\
\text { canine }\end{array}$} & Oonventional extrusion & 30 & 48.3 & 23.7 \\
\cline { 2 - 5 } & Optimized extrusion & 40 & 44.8 & 29.3 \\
\cline { 2 - 5 } & Optimized extrusion & 19 & 46.6 & 28.0 \\
\cline { 2 - 5 } & Conventional rotation & 17 & 57.9 & 35.7 \\
\hline \multirow{4}{*}{$\begin{array}{c}\text { Maxillary } \\
\text { premolar }\end{array}$} & Optimized rotation & 38 & 65.9 & 29.8 \\
\hline \multirow{2}{*}{$\begin{array}{c}\text { Mandibular } \\
\text { incisor }\end{array}$} & Conventional rotation & 10 & 48.1 & 23.9 \\
\cline { 2 - 5 } & Optimized rotation & 36 & 72.8 & 23.6 \\
\cline { 2 - 5 } & Oonventional extrusion & 7 & 27.7 & 33.3 \\
\hline \multirow{4}{*}{$\begin{array}{c}\text { Mandibular } \\
\text { canine }\end{array}$} & Conventional extrusion & 5 & 64.8 & 24.3 \\
\cline { 2 - 5 } & Optimized extrusion & 2 & 16.1 & 18.8 \\
\cline { 2 - 5 } & Conventional rotation & 19 & 54.5 & 53.8 \\
\cline { 2 - 5 } & Optimized rotation & 35 & 60.5 & 25.0 \\
\hline \multirow{2}{*}{$\begin{array}{c}\text { Mandibular } \\
\text { premolar }\end{array}$} & Conventional rotation & 26 & 68.0 & 25.9 \\
\cline { 2 - 5 } & Optimized rotation & 54 & 58.6 & 28.9 \\
\hline
\end{tabular}

Mean accuracy for each tooth type is displayed in Table 8 for rotation and Table 9 for extrusion. Two one-way ANOVAs were used to compare accuracy by tooth types for both movements (Table 10). For rotation, there was no significant difference between tooth types $(P>0.05)$. However, for extrusion, there was a significant difference between maxillary central incisors and maxillary canines, and between maxillary central incisors and mandibular canines. The maxillary central incisor had the highest mean accuracy for extrusion. These significant differences were determined using a Bonferroni post-hoc test. 
Table 8. Mean Accuracy by Tooth Type: Rotation

\begin{tabular}{|c|c|c|c|}
\hline Tooth & n & Mean Accuracy (\%) & SD \\
\hline Maxillary canine & 55 & 63.4 & 25.2 \\
\hline Maxillary premolar & 46 & 67.4 & 25.5 \\
\hline Mandibular canine & 54 & 65.4 & 25.6 \\
\hline Mandibular premolar & 80 & 59.3 & 28.7 \\
\hline
\end{tabular}

Table 9. Mean Accuracy by Tooth Type: Extrusion

\begin{tabular}{|c|c|c|c|}
\hline Tooth & $\mathbf{n}$ & Mean Accuracy (\%) & SD \\
\hline Maxillary central incisor & 22 & 66.3 & 22.6 \\
\hline Maxillary lateral incisor & 70 & 46.3 & 26.9 \\
\hline Maxillary canine & 32 & 41.7 & 32.9 \\
\hline Mandibular incisor & 16 & 48.5 & 33.5 \\
\hline Mandibular canine & 7 & 27.1 & 32.7 \\
\hline
\end{tabular}

Table 10. ANOVA Comparing Mean Accuracy by Tooth Type

\begin{tabular}{|c|c|c|c|c|}
\hline Tooth Movement & df & Mean Square (\%) & F & $P$-value \\
\hline Rotation & 3 & 769.0 & 1.1 & 0.3546 \\
\hline Extrusion * & 4 & 2972.5 & 3.6 & 0.0080 \\
\hline
\end{tabular}

$* P<0.05$

\section{E. Optimized vs. Conventional Attachments}

The results of two independent $t$-tests comparing absolute mean differences of teeth with optimized and conventional attachments can be seen in Table 11. Mean difference between the two attachment types was calculated by subtracting mean optimized values from mean conventional values. Mean difference for rotation was $1.61^{\circ}$, which was not statistically significant $(P>0.05)$. Mean difference for extrusion was $0.14 \mathrm{~mm}$ and was also found to not reach statistical significance $(P>0.05)$. Teeth with conventional attachments had a larger mean discrepancy. 
Table 11. Optimized Attachments vs. Conventional Attachments ( ${ }^{\circ}$ and $\left.\mathrm{mm}\right)$

\begin{tabular}{|c|c|c|c|c|c|c|}
\hline \multirow{2}{*}{ Tooth Movement } & \multirow{2}{*}{$\mathbf{n}$} & \multirow{2}{*}{$\begin{array}{c}\text { Abs Mean Difference } \\
\text { (|Conventional-Optimized } \mid \text { ) }\end{array}$} & \multirow{2}{*}{ SD } & \multicolumn{2}{|c|}{$95 \% \mathrm{CI}$} & \multirow{2}{*}{$P$-value } \\
\hline & & & & Lower & Upper & \\
\hline Rotation ( ${ }^{\circ}$ ) & 235 & 1.61 & 6.11 & 0.10 & 3.32 & 0.0638 \\
\hline Extrusion (mm) & 147 & 0.14 & 0.42 & 0.00 & 0.27 & 0.0523 \\
\hline
\end{tabular}

Table 12 lists the percent accuracy of optimized and conventional attachments for both tooth movements. For optimized rotation, mean accuracy was $65.5 \%$, while for conventional rotation it was $58.2 \%$. The difference between the two was not statistically significant $(P>0.05)$, as seen in Table 13. For optimized extrusion, mean accuracy was $49.6 \%$, while for conventional extrusion it was $45.2 \%$. The mean difference between optimized and conventional attachments was also not statistically significant for extrusion $(P>0.05)$.

Table 12. Mean Accuracy by Attachment Type and Tooth Movement

\begin{tabular}{|c|c|c|c|}
\hline Attachment and Tooth Movement & $\mathbf{n}$ & Mean Accuracy (\%) & SD \\
\hline Optimized Rotation & 163 & 65.5 & 25.9 \\
\hline Conventional Rotation & 72 & 58.2 & 27.6 \\
\hline Optimized Extrusion & 81 & 49.6 & 30.5 \\
\hline Conventional Extrusion & 66 & 45.2 & 28.9 \\
\hline
\end{tabular}

Table 13. Optimized Attachments vs. Conventional Attachments (\%)

\begin{tabular}{|c|c|c|c|c|c|c|}
\hline \multirow{2}{*}{$\begin{array}{c}\text { Tooth } \\
\text { Movement }\end{array}$} & \multirow{2}{*}{$\mathbf{n}$} & \multirow{2}{*}{$\begin{array}{c}\text { Mean \% Difference } \\
\text { (Optimized - Conventional) }\end{array}$} & \multirow{2}{*}{ SD } & \multicolumn{2}{|c|}{$95 \%$ CI } & \multirow{2}{*}{$P$-value } \\
\hline & & & & Lower & Upper & \\
\hline Rotation & 235 & 7.3 & 26.4 & -0.1 & 14.6 & 0.0533 \\
\hline Extrusion & 147 & 4.3 & 29.8 & -5.4 & 14.1 & 0.3819 \\
\hline
\end{tabular}




\section{F. Interproximal Reduction (IPR) and Spacing}

When comparing teeth that had spacing or IPR to those without, mean accuracy for both conditions was about 57\% (Table 14). Using three independent $t$-tests (Table 15), it was found that, for all teeth, IPR or spacing only slightly improved accuracy by $0.2 \%$, and this did not reach statistical significance $(P>0.05)$. For canines and premolars that had IPR or spacing and underwent rotation, the percent accuracy actually decreased by $3 \%$. For incisor or canine extrusion, IPR or spacing improved accuracy by $1.2 \%$.

None of these differences were statistically significant $(P>0.05)$.

Table 14. Mean Accuracy by Interproximal Condition

\begin{tabular}{|c|c|c|c|}
\hline Interproximal Condition & $\mathbf{n}$ & Mean Accuracy (\%) & SD \\
\hline IPR or spacing & 61 & 57.4 & 28.6 \\
\hline No IPR or spacing & 321 & 57.2 & 28.9 \\
\hline
\end{tabular}

Table 15. IPR or spacing vs. no IPR or spacing

\begin{tabular}{|c|c|c|c|c|c|c|}
\hline \multirow{2}{*}{ Tooth Movement } & $\mathbf{n}$ & $\begin{array}{c}\text { Mean Difference (\%) } \\
\text { (IPR or spacing- } \\
\text { no IPR or spacing) }\end{array}$ & $\mathbf{S D}$ & \multicolumn{2}{|c|}{$\mathbf{9 5 \%}$ CI } & \multirow{2}{*}{ P-value } \\
\hline Rotation & 235 & -3.0 & 26.6 & -11.8 & 5.8 & 0.503 \\
\hline Extrusion & 147 & 1.2 & 29.9 & -14.0 & 16.4 & 0.8777 \\
\hline Total & 382 & 0.2 & 28.9 & -7.7 & 8.2 & 0.9528 \\
\hline
\end{tabular}




\section{CHAPTER VI: DISCUSSION}

\section{A. Study Aim}

A 2018 systematic review assessing the clinical effectiveness of Invisalign found that the clear aligner system can predictably level, level, tip, and derotate anterior teeth, but not canines and premolars. Other limitations included bodily tooth movement and extrusion of incisors (Papadimitriou et al.). The present study focused on intra-arch measurements of two tooth movements reported to be the least accurate with Invisalign rotation of canines and premolars, and extrusion of incisors and canines. These movements were also chosen since they have specific optimized attachments available to compare to conventional ones. No published study to date has compared the efficacy of Invisalign's two attachment types for any tooth movement.

\section{B. Sample}

Although cases from two separate clinicians were used, both were orthodontists who had several years of experience with the Invisalign system. Furthermore, due to the strict inclusion and exclusion criteria, cases included in this study were typically Class I malocclusions, where the clinicians simply had a preference of using optimized or conventional attachments to help resolve crowding or extrude teeth. Though open bites were commonly encountered, it was difficult to find cases that had anterior extrusion planned without concomitant planned intrusion of posterior teeth. Overall, the study sample was representative of the general orthodontic population, as the majority of predicted tooth movements were mild rotations $<45^{\circ}$ and mild extrusion $<2.5 \mathrm{~mm}$. 


\section{Results Analysis}

The overall mean accuracy of tooth movements was found to be $57.2 \%$, but it must be noted that only rotation and extrusion were analyzed in cases without any treatment adjuncts (i.e. elastics). Therefore, these results should not be generalized for all patients treated with Invisalign. This study does, however, provide useful information on accuracy of these two difficult tooth movements and of the attachment types used over a series of aligners.

In general, it was found that for all tooth movements, predicted values were higher than achieved values, and these results were statistically significant. For rotation, mean difference between predicted and achieved values ranged from $4.01^{\circ}$ to $6.01^{\circ}$ for optimized and conventional attachments, respectively. A previous study by Kravitz et al. used $15^{\circ}$ as a clinically discernable amount of malrotation ("How well does Invisalign work?"). Based on this cut-off value, these discrepancies were not clinically significant. However, clinicians may still elect to overcorrect canine and premolar rotations by $4^{\circ}$ to $6^{\circ}$ based on the findings of this study. For extrusion, mean difference ranged from 0.40 $\mathrm{mm}$ to $0.62 \mathrm{~mm}$ for optimized and conventional attachments, respectively. Since these discrepancies were above the $0.2 \mathrm{~mm}$ limit of resolution of the human eye (Bille et al.) and since orthodontists are trained to focus on anterior microesthetics during the "finishing" stages of treatment, they are to be considered clinically significant. Clinicians should, therefore, plan to overcorrect anterior extrusion by $0.4 \mathrm{~mm}$ to $0.6 \mathrm{~mm}$.

Kravitz et al. found that the least accurate tooth movement with Invisalign was extrusion of incisors $(29.6 \%)$ and that, for rotation, the least accurate tooth was the canine $(35.8 \%)$. He also reported that for rotations greater than $15^{\circ}$, accuracy significantly fell 
by up to $52.5 \%$ (“How well does Invisalign work?"). Other studies reported $29.1 \%$ to 49.7\% accuracy for canine and premolar derotation (Simon et al). The current study found a mean accuracy of $63.2 \%$ for these rotational movements and $47.6 \%$ for extrusion of anterior teeth. These findings are higher than previous studies show. Differences may be due several factors, including but not limited to introduction of SmartTrack material in 2014, smaller sample size in previous studies, variable patient compliance, frequency of aligner switches, or the presence or absence of attachments.

In the current study, the least accurate tooth movement was extrusion of the mandibular canine with a conventional attachment (16.1\%). The most accurate tooth movement was extrusion of maxillary central incisors with a conventional attachment (73.9\%), followed closely by rotation of maxillary premolars with an optimized attachment (72.8\%). When comparing optimized and conventional attachments, mean difference for rotation was $1.61^{\circ}$ or $7.3 \%$. For extrusion, mean difference was $0.14 \mathrm{~mm}$ or $4.3 \%$. Though optimized attachments had a higher mean accuracy than conventional attachments for both movements, these differences were neither statistically nor clinically significant.

Kravitz et al. found that the presence of attachments did not significantly improve the accuracy of canine rotation ("Influence of attachments and interproximal reduction"). However, the most common attachment in that study was the vertical ellipsoid, which is rarely used today, and which has been replaced by optimized or rectangular conventional attachments. According to the literature, derotation of a cylindrical tooth is difficult since aligners tend to lose anchorage and slip off due to a lack of undercuts and a round tooth 
shape (Simon et al.). It would make sense that attachments would provide some grip and surface area for forces to be applied, as long as the aligners fit well.

In this study, IPR and spacing were grouped together since both conditions would, in theory, reduce friction between teeth during movement. Kravitz et al. reported that IPR did not significantly improve the accuracy of canine rotations ("Influence of attachments and interproximal reduction"). The findings from the current study also found that there was no significant improvement in accuracy when IPR was used or when spacing was present, with both the presence and absence of these conditions reaching $57 \%$ accuracy. This may be because of the small sample size of teeth that had IPR or spacing (16\%). Additionally, IPR is most commonly performed on lower anterior teeth to help resolve crowding, and anterior rotations were not evaluated in this study.

\section{Limitations}

While intra-examiner reliability scores for measurements was high, error in model superimposition was not accounted for. The study sample included 37 patients under the age of 18 years old. Of these, 16 patients were between the ages of 11 and 14 years who were growing and likely had second molars erupting. Both of these patient factors may have affected digital model superimposition on posterior teeth. Posterior teeth with no planned movement were chosen to superimpose on since no other stable landmarks (i.e. palatal rugae or dental implants) were available. Palatal rugae could not be used since the predicted models exported from ClinCheck only illustrate the dentition and attached gingiva.

Even though posterior teeth that had no planned movement were used for superimpositions, intrusion of posterior teeth between 0.25 to $0.5 \mathrm{~mm}$ is often observed 
due to plastic thickness of the appliance (Phan and Ling). This would have affected vertical measurements the most. Lateral cephalograms were not used to evaluate any amount of posterior intrusion, since 1) if available, they were taken pre- and posttreatment, but these post-treatment radiographs did not correspond to the same timepoints as refinement scans that were used for "final" digital models, and 2) this small amount of posterior tooth movement would be difficult to accurately measure after already manually superimposing radiographs. For these reasons, "control" teeth were used to calculate achieved extrusion. Although this likely improved the accuracy of measurements, "control" teeth that had no predicted movement may have still moved in the vertical dimension, especially if they had an attachment on them for planned movement in a different plane of space or simply for support of the aligner.

Even though the minimum values for Invisalign's software to apply optimized rotation and extrusion attachments are $5^{\circ}$ and $0.5 \mathrm{~mm}$, respectively, the lowest predicted values in this study were $2.2^{\circ}$ and $0.18 \mathrm{~mm}$. This may be due to a difference between Invisalign's ToothMeasure software in ClinCheck and the superimpositions and measurements performed in the 3D Slicer CMF program. It was not possible to crosscheck the mild predicted values measured in this study with what was predicted by Invisalign, as older versions of ClinCheck before early 2018 did not have a Tooth Movements Table that reports these values.

In addition to potential software discrepancies, other limitations of a retrospective study included inability to account for certain variables, such as conventional attachment features, simultaneous tooth movements, periodontal support, and patient compliance. Location of placement, size, orientation, and beveling of conventional attachments was 
not considered in this study. Although having an optimized rotation and extrusion attachment applied means that those were the primary movements for a specific tooth, it is possible that other small simultaneous movements were occurring in different planes of space. Furthermore, though most of the cases used initial scans, this study also used some patients' refinement scans, meaning they already had prior tooth movement. Any existing mobility and altered periodontal support may have influenced results, along with general periodontal status and bone density ranging from patient to patient.

\section{E. Future Direction}

Future studies should be prospective and consider using 2-D lateral cephalograms or 3-D cone beam computed tomography (CBCT) imaging to evaluate tooth movement rather than superimposing on posterior teeth, which may inadvertently move during orthodontic treatment. Additionally, other attachment variables previously mentioned should be considered and more types of tooth movement assessed to compare the efficacy of conventional and optimized attachments. 


\section{CHAPTER VII: CONCLUSION}

Overall, mean accuracy of both tooth movements (rotation and extrusion combined) was found to be $57.2 \%$. This study showed that rotations of canines and premolars can be accomplished using the Invisalign system with a $63.2 \%$ mean accuracy, and extrusion of incisors and canines can be achieved with a mean accuracy of $47.6 \%$. The most accurate tooth movement was extrusion of maxillary central incisors with a conventional attachment (73.9\%), followed closely by rotation of maxillary premolars with an optimized attachment (72.8\%). The least accurate tooth movement was extrusion of mandibular canines with an optimized attachment (16.1\%). There was a significant difference between accuracy of maxillary central incisor extrusion and both maxillary and mandibular canine extrusion.

For both rotation and extrusion, the mean difference values between predicted and achieved movements was highly statistically significant. For extrusion, this was also clinically significant (mean difference $>0.2 \mathrm{~mm}$ ), but for rotation, it was not (mean difference $<15^{\circ}$ ). Clinicians may consider overcorrecting rotations by $4^{\circ}$ to $6^{\circ}$ to improve accuracy, and should overcorrect extrusion by $0.4 \mathrm{~mm}$ to $0.6 \mathrm{~mm}$. IPR or spacing did not significantly improve accuracy for rotations of rounded teeth and for extrusion of anterior teeth. A larger sample size for teeth with these conditions is needed for more definitive conclusions.

Mean difference in accuracy between teeth with optimized and conventional attachments was neither clinically nor statistically significant for both rotation and extrusion, thus failing to reject both null hypotheses. These results can be of value to any clinician offering clear aligner therapy, as it can help build confidence in his or her 
treatment plan. Users should be prepared for mid-course correction and consider overcorrection, especially for extrusion of anterior teeth. With more companies offering clear aligners and with the emergence of in-house 3-D printing, one can feel confident in knowing that conventional attachment types may be just as effective as Invisalign's proprietary optimized attachments for rotations of canines and premolars and for extrusion of incisors and canines. 


\section{REFERENCES}

Align Technology, Inc. "Invisalign Tooth Movement Assessment Overview." Invisalign Online Academy, Santa Clara, CA, 2013 (learn.invisalign.com). Accessed 16 June 2019.

Align Technology, Inc. "Living with Invisalign Clear Aligners." Invisalign, Santa Clara, CA (www.invisalign.com/how-invisalign-works/living-with-invisalign). Accessed 16 June 2019.

Align Technology, Inc. "SmartForce Features." Invisalign Online Academy, Santa Clara, CA (learn.invisalign.com/smartforce). Accessed 16 June 2019.

Align, Technology, Inc. "The Invisalign Difference.” Invisalign, Santa Clara, CA (www.invisalign.com/the-invisalign-difference). Accessed 16 June 2019.

Align, Technology, Inc. "Tips for Success with Invisalign Optimized Attachments." Invisalign Online Academy, Santa Clara, CA, 2011. (learn.invisalign.com/attachments/bonding/procedure/tips_tricks). Accessed 1 Nov. 2018.

Bille Josef F, et al. "Optical Quality of the Human Eye: The Quest for Perfect Vision." Aberration-Free Refractive Surgery: New Frontiers in Vision, edited by Bille, Harner, Loesel, e-book, Springer-Verlag, Heidelberg, 2003, pp. 25-46.

Buschang, Peter $\mathrm{H}$, et al. "Predicted and actual end-of-treatment occlusion produced with aligner therapy." Angle Orthodontist, vol. 85, no. 5, Nov. 2014, pp. 723-727.

Charalampakis, Orfeas et al. "Accuracy of clear aligners: A retrospective study of patients who needed refinement." American Journal of Orthodontics and Dentofacial Orthopedics, vol. 154, no. 1, July 2018, pp. 47-54.

Condo, Roberta, et al. "Mechanical properties of 'two generations' of teeth aligners: Change analysis during oral permanence." Dental Materials Journal, vol. 37, no. 5, Sept. 2018, pp. 835-842.

Graber, Lee W, et al. Orthodontic: Current Principles and Techniques. $6^{\text {th }}$ ed., e-book, St. Louis, MO, Elsevier, 2017.

Kravitz, Neal D, et al. "How well does Invisalign work? A prospective clinical study evaluating the efficacy of tooth movement with Invisalign." American Journal of Orthodontics and Dentofacial Orthopedics, vol. 135, no. 1, Jan. 2009, pp. 27-35.

Kravitz, Neal D, et al. "Influence of attachments and interproximal reduction on the 
accuracy of canine rotation with Invisalign. A prospective clinical study." Angle Orthodontist, vol. 78, no. 4, July 2008, pp. 682-687.

Krieger, Elena, et al. "Accuracy of Invisalign treatments in the anterior tooth region: First results." Journal of Orofacial Orthopedics, vol. 72, no. 2, March 2011, pp. 141149.

Krieger, Elena, et al. "Invisalign treatment in the anterior tooth region: Were the predicted tooth movements achieved?" Journal of Orofacial Orthopedics, vol. 73, no. 5, Sept. 2012, pp. 365-376.

Papadimitriou, Aikaterini, et al. "Clinical effectiveness of Invisalign orthodontic treatment: a systematic review." Progress in Orthodontics, vol. 19, no. 37, Sept. 2018, pp. 1-24.

Phan, Xiem and Paul H. Ling. "Clinical Limitations of Invisalign." Journal of the Canadian Dental Association, vol. 73, no. 3, April 2007, pp. 263-266.

Proffit, William R, et al. Contemporary Orthodontics. $5^{\text {th }}$ ed., e-book, St. Louis, MO, Elsevier, 2012.

Simon, Mareike, et al. "Forces and moments generated by removable thermoplastic aligners: Incisor torque, premolar derotation, and molar distalization." American Journal of Orthodontics and Dentofacial Orthopedics, vol. 145, no. 6, June 2014, pp. 728-736.

Simon, Mareike, et al. "Treatment outcome and efficacy of an aligner technique regarding incisor torque, premolar derotation, and molar distalization." BioMedCentral Oral Health, vol. 14, no. 68, June 2014, pp. 1-7.

Ziuchkovski, James P, et al. "Assessment of perceived orthodontic appliance attractiveness." American Journal of Orthodontics and Dentofacial Orthopedics, vol. 133, no. 4, supplement 1, April 2008, pp. S68-78. 